EPiC Series in Engineering
Volume 2, 2018, Pages 183-193
SUMO 2018- Simulating Autonomous
and Intermodal Transport Systems

\title{
Simulation of Autonomous RoboShuttles in Shared Space
}

\author{
Rainer Gasper ${ }^{1}$, Stephan Beutelschie $\aleph^{2}$, Mario Krumnow ${ }^{2}$, Levente Simon ${ }^{1}$, \\ Zoltan Baksa ${ }^{1}$, Jochen Schwarzer ${ }^{1}$ \\ ${ }^{1}$ Robert Bosch GmbH \\ ${ }^{2}$ TU Dresden \\ rainer.gasper@de.bosch.com, stephan.beutelschiess@gmail.com, \\ mario.krumnow@tu-dresden.de, levente.simon@hu.bosch.com, \\ zoltan.baksa@hu.bosch.com, jochen.schwarzer@de.bosch.com
}

\begin{abstract}
In the mobility sector, autonomous driving will become more and more part of our daily life. Most of all, in public transportation the research to exploit the new possibilities of autonomous driving has increased drastically. But the problem of the last mile is still unsolved, for example. The last mile is the problem to transport people from a transportation network (examples of endpoints are parking lots or bus station) to their final destination. A promising solution to this problem are autonomous RoboShuttles. Because of their low velocity, they can operate in the shared space where pedestrians and vehicles share the same traffic area. Therefore, in a shared space the interaction between them is greater than under normal traffic conditions, where the traffic flows are separated as much as possible. Through this higher interaction, new requirements on the autonomous vehicle arise. To explore the new requirements and to understand the interaction of a RoboShuttle in a shared space, a simulation scenario in SUMO is set up.
\end{abstract}

\section{Introduction}

The automation of vehicles is one of the big challenges not only for the private transport with personally owned cars, but also for the public transportation sector. The research in the public transportation area with autonomous vehicles as topic increased drastically in the last few years, see for example (CITYMOBIL2, 2016) (IVI, 2017) (Marshall, 2017) (Tor, 2017). But the problem of the last mile is still unsolved, for example. The last mile is the problem to transport people from a transportation network (examples of endpoints are parking lots or bus station) to their final destination. To solve this problem autonomous RoboShuttles are very promising. A RoboShuttle has a transportation capacity between 4 and 12 persons. Normally, the maximum velocity is in the range of $20-40 \mathrm{~km}$ per hour, depending on the area they operate. Because of their low velocity, they can operate in the shared space where pedestrians and vehicles share the same traffic area. Therefore, the interaction between vehicles 
and pedestrian in a shared space is greater than under normal traffic conditions, where the traffic flows are separated as much as possible. But through this higher interaction new requirements on the autonomous vehicle arise.

There are already RoboShuttles on the market available. For example AURO (AURO, 2017), EasyMile (EASYMILE, 2017), and WAYMO (WAYMO, 2017) sell RoboShuttles. Also, the "Deutsche Bahn" uses already RoboShuttles (Deutsche Bahn AG, 2017).

To explore the new requirements and to understand the interaction of a RoboShuttle in a shared space, a simulation scenario in SUMO (Daniel Krajzewicz, 2012) is set up. As an example for a shared space and the problem of the last mile, the research campus of the Robert Bosch GmbH in Renningen is considered. For the simulation, a traffic demand model was developed and the simulation of pedestrians and vehicles on the campus will be described. Also, an operating strategy to minimize the travel time of the pedestrians will be presented. The article is organized as follows. First, a description of the simulation scenario is given. That includes the network and the traffic demand on the campus. After that, the operation strategy will be described. At the end of the article, simulation results will be shown and discussed.

\section{Modelling of the RoboShuttle and the Shared Space with Pedestrians}

The considered shared space is the research campus of the Robert Bosch GmbH in Renningen. It is a closed area where the vehicle traffic consists only of RoboShuttles. The RoboShuttles have to share the traffic area with pedestrians. To simulate the scenario of a shared space, SUMO has to take into account the interaction of pedestrians and vehicles.

Pedestrians and vehicles differ in their dynamic behavior. For example, the walk velocity of a pedestrian is much lower than the vehicle velocity. But the pedestrian can turn on the spot, which is normally not possible for a vehicle. The main differences of the behaviors in SUMO of the different models are summarized in Table 1.

In the following, the pedestrian and vehicle models are described. After that, a description of the shared space network is given.

\begin{tabular}{|l|c|c|}
\hline Behavior & Pedestrian & Vehicle \\
\hline Moving on edge & $\begin{array}{c}\text { Bidirectional movement on } \\
\text { edge possible }\end{array}$ & $\begin{array}{c}\text { movement on edge only in } \\
\text { positive direction possible }\end{array}$ \\
\hline Vehicle as transportation & $\begin{array}{l}\text { Multi modal travelling } \\
\text { possible by entering vehicles }\end{array}$ & $\begin{array}{c}\text { No transportation of } \\
\text { vehicles in vehicles }\end{array}$ \\
\hline Control with TraCI & $\begin{array}{c}\text { Only restricted } \\
\text { Strong controllability } \\
\text { Rplit in stages }\end{array}$ & $\begin{array}{l}\text { The route defined by the } \\
\text { edges }\end{array}$ \\
\hline Velocity & $\begin{array}{l}\text { Change in velocity is } \\
\text { instantaneous, without } \\
\text { considering a maximal } \\
\text { acceleration }\end{array}$ & $\begin{array}{l}\text { Velocity is defined by the } \\
\text { collowing model }\end{array}$ \\
\hline
\end{tabular}

Table 1: comparison of vehicle and pedestrians behavior in SUMO 


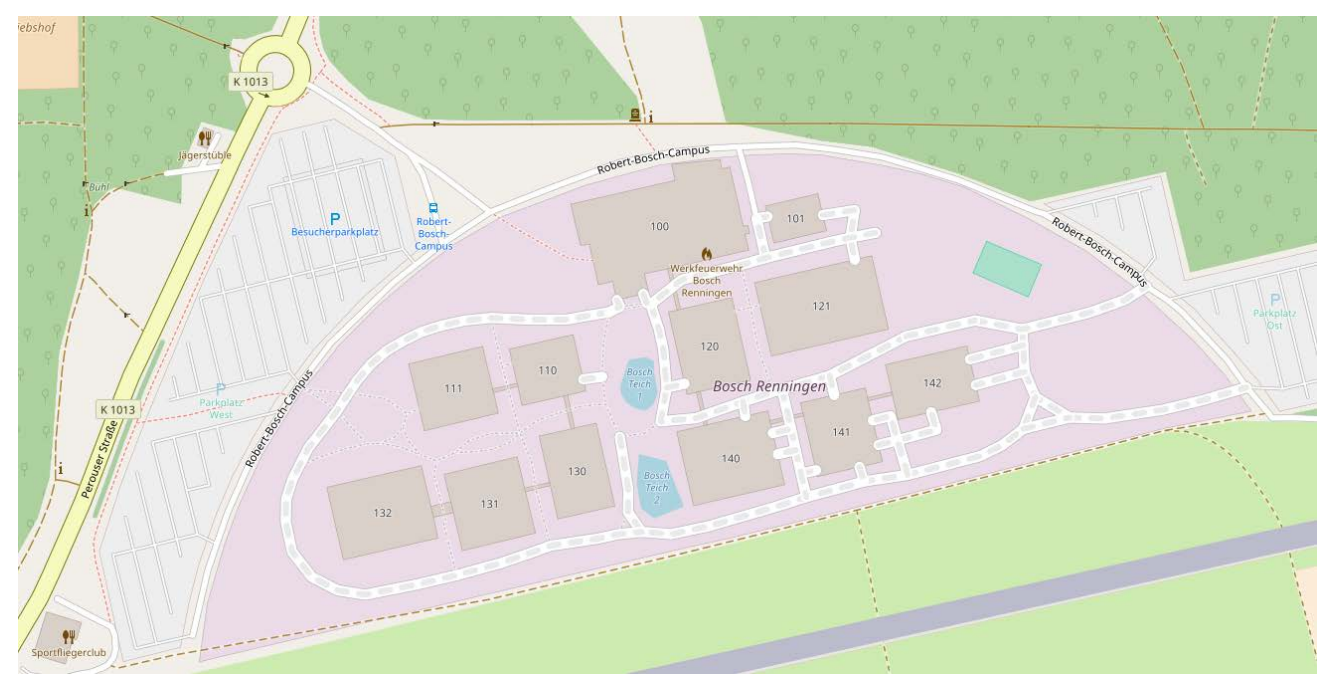

Figure 1: Research Campus Renningen in openstreetmap.org

\subsection{RoboShuttle}

The car following model and the lane change model defines the behavior of the vehicle. For the car following model the Krauss-model is used. The parameter sigma, which describes the driver imperfection, is set to zero because the RoboShuttle is automated. For autonomous shuttles the reaction time is assumed to be $0.1 \mathrm{sec}$. and the parameter tau is also set to $0.1 \mathrm{sec}$. because of the simulation step size of $0.1 \mathrm{sec}$.

As lane change model the SL2015 model in SUMO is used. The lane change model is responsible for the lateral movement. The maximum lateral acceleration per second lcAccelLat is set to $0.8 \frac{\mathrm{m}}{\mathrm{s}^{2}}$ to meet the comfort requirements. The maximum velocity is $20 \mathrm{~km}$ per hour and the capacity is 12 passengers.

\subsection{Pedestrian}

The model of a pedestrian is different from the vehicle model in SUMO. The trips for pedestrians are split in stages. The three available stages are the walkingStage, where the pedestrian only walks, the drivingStage, where the pedestrian rides with the shuttle e.g., and the waitingStage, where the pedestrian stands still and waits.

The pedestrian is labeled with the ID "personID". For the pedestrians the default values of the DEFAULT_PEDTYPE in SUMO are used because the parameters describe a typical pedestrian behavior. It is assumed that pedestrians don't walk with constant velocity and therefore the default value 0.2 is used for the option pedestrian.striping.dawdling.

\subsection{Interaction between vehicles and pedestrians}

In SUMO it is possible to model a multi modal trip of pedestrians. Pedestrians can board a vehicle, drive around as passengers, and can exit the vehicle at a stop. The vehicles can be busses, passenger 


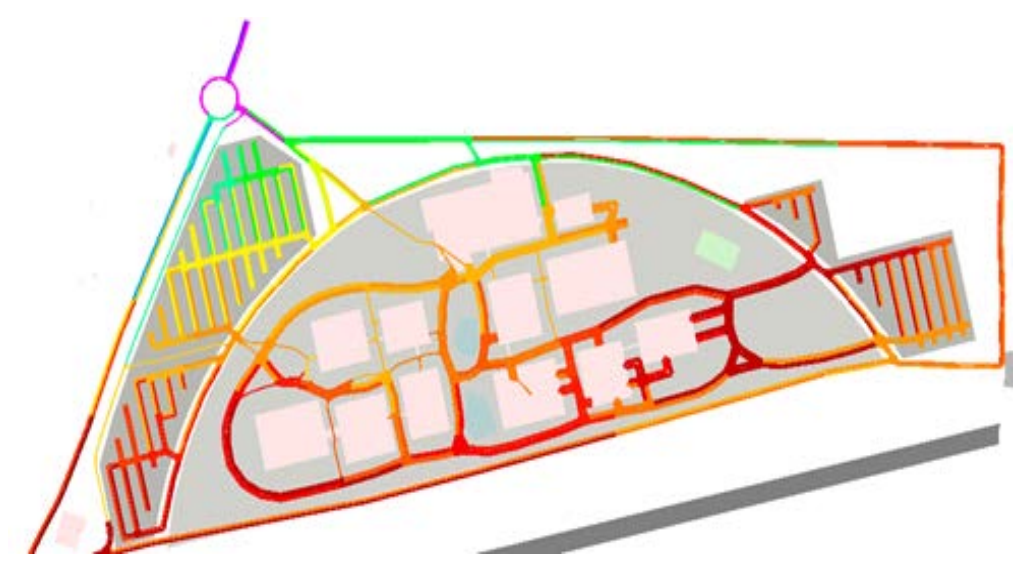

Figure 2: Network of the research campus with elevation data in SUMO

cars and so on. To enable this behavior a ride stage has to be defined in the trip definition, or at run time a driving stage has to be send to the simulation via TRACI.

In the following an example for a pedestrian trip is given. The pedestrian is set at time 0 into the simulation. He walks on the edges "a, b, c". Existing pavements on the edges will be used. The pedestrian sends a request to the operation strategy, which checks the requirements and after a positive check orders the nearest RoboShuttle to the edge c. The pedestrian enters the RoboShuttle at the stop. The shuttle drives with the passengers on board to the desired edge. How the destination edge of the shuttle is determined is described in the operation strategy section.

The stop time of the shuttles is as short as possible. In (A. Neumann, 2014) the boarding and exit times in public transportation are evaluated. The boarding takes longer than the exit because in the considered public busses the boarding was only possible at the front door, where also the bus tickets are sold. In case of the RoboShuttle the boarding and exit times are assumed to be $1.5 \mathrm{sec}$. for both, because no tickets have to be bought and the number of entries and exits is equal.

In the simulation the pedestrians on a lane are detected so that collisions are avoided. Furthermore, with the available sub lane model in SUMO, it is possible that the shuttle can overtake a pedestrian without changing the lane if enough space exists on the lane.

\subsection{Network modelling of the research campus in Renningen}

The modelling of the network for the traffic flow simulation can be done from scratch or by using map data from openstreetmap.org (OPENSTREETMAP, 2017). Because the research campus in Renningen is available in openstreetmap.org, see Figure 1, and to build up the network from scratch is time consuming, the data for the network have been downloaded from openstreetmap.org. After the download, the map was refined in JOSM to make the map more suitable for the simulation. Also the tool osmfilter (OSMFILTER, 2017) was used to filter elements by tag from the map data. After refining, the network was processed with netconvert to obtain a SUMO - network. 


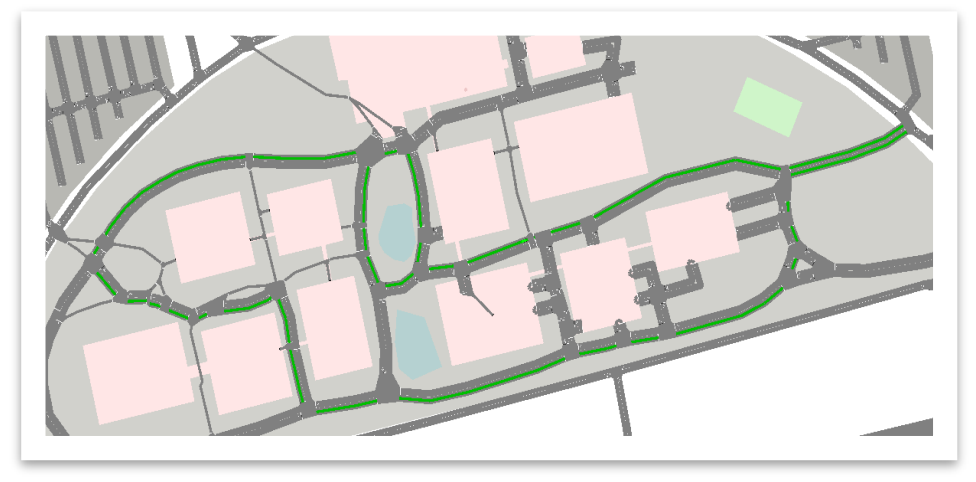

Figure 3: route in green for the RoboShuttles on the research campus

The network was also augmented with the elevation data of the research campus. As source for the elevation data the geotiff data from the NASA SRTM mission have been used (NASA). The elevation data associated with the nodes were identified and the elevation data on the edges were interpolated linearly between the nodes. The network of the research campus in Renningen with elevation data is shown in Figure 2. Between the lowest point with $448 \mathrm{~m}$ altitude (in red) and the highest point with 452 $\mathrm{m}$ altitude (in orange) on the route, the difference is only $4 \mathrm{~m}$, which can be neglected for the energy consumption of the RoboShuttle. The highest point on the campus has an altitude of $462 \mathrm{~m}$ (in green).

\section{Traffic Demand Modelling}

The modelling of traffic demand in the shared space is split into the pedestrian traffic demand and the vehicle traffic demand of the RoboShuttles. Whereby in the shared space the main focus is on the pedestrian traffic demand.

\subsection{Pedestrian traffic demand}

The RoboShuttle has to transport the pedestrians from their current position to their final destinations. The main point of interests are the entries to the research campus, the working places and the cafeteria. A typical pedestrian traffic flows in the morning from the entries to the working places, at lunchtime from the working places to cafeteria and vice versa, and in the evening from the working places back to the entries where the employees leave the research campus.

To model these traffic flows of pedestrians the poison distribution is used,

$$
P(X=x)=\frac{\lambda^{x}}{x !} e^{-\lambda}
$$

where $\lambda$ is the expectation value of the distribution and $x \in Z$. The parametrization of the distribution was done to meet the incoming and outgoing flows of the employees at the entries in combination of the working places in the buildings. In addition, the number of lunches was used to estimate the number of visitors at lunchtime in the cafeteria. 


\subsection{Vehicle traffic demand}

Because the considered traffic area is a closed traffic area, there is only the vehicle traffic generated by the RoboShuttles. The RoboShuttles drive on a defined route, see Figure 3. The vehicles are inserted in the simulation via TraCI and are driving on the given route. Therefore, no further consideration of the vehicle traffic demand is necessary. The number of RoboShuttles in the shared space is varied in the simulation. The pedestrians define the stops on the campus.

\section{Operation Strategy}

The operation strategy has to improve the situation for the pedestrians. Therefore, the travel time of the pedestrians has to be minimized. The operation strategy is shown in Figure 4.

The pedestrian sends a request to the operation strategy. With the actual position, the time to destination is analyzed. In the first step, the operation strategy has to check if there is at least one shuttle with a free seat available. Then, the time to walk to the destination $t$ __walk is determined with a mean velocity of $1.2 \mathrm{~m} / \mathrm{s}$ for pedestrians. The velocity is a conservative estimation so that the travel time is overestimated.

After that, a stop for the shuttle is determined where the pedestrian can enter the shuttle. The stop has to meet the conditions that it is on the route of the shuttle and is the nearest to the actual position of the pedestrian. With the distance of the pedestrian to the stop and the pedestrian velocity, the time to walk to the stop tt_entry_Pers can be calculated.

To calculate the travel time tt_entry_Shuttle of the shuttle to the stop, the distance of the shuttle depending on the known route is determined.

A requirement is that the pedestrian has to be first on the stop to avoid unnecessary waiting time of the shuttle. Therefore, the travel time of the shuttle to the stop has to be greater than the walking time of the pedestrian. The shuttle velocity to calculate the travel time is set to the maximum velocity 5.55 $\mathrm{m} / \mathrm{s}$. the travel time is therefore underestimated and the constraint $t$ _entry_Pers $<t t \_e n t r y \_S h u t t l e$ leads too often to a negative assessment. To compensate this underestimation, the pedestrian velocity is assumed to be $1.2 \mathrm{~m} / \mathrm{s}$, which is low, as mentioned above. With the maximal shuttle velocity of $5.5 \mathrm{~m} / \mathrm{s}$ and a low pedestrian velocity of $1.2 \mathrm{~m} / \mathrm{s}$ it is guaranteed that the pedestrian will be the first at the stop.

Is the walking time $t$ __walk less than the travel time to the stop $t$ _entry_Shuttle the minimization is terminated.

In the next step of the operation strategy, the exit point is determined so that the multi modal travel time $t$ __multimodal is minimized. For the exit point, all edges on the shuttle route are considered. For each edge, the driving time with the shuttle from the entry to the exit point and the walking time from the exit point to the destination have to be determined. The optimal exit point is not the edge with the minimal distance as in the case for the entry point, but the exit point is determined by minimizing the sum of the walking and driving time. 


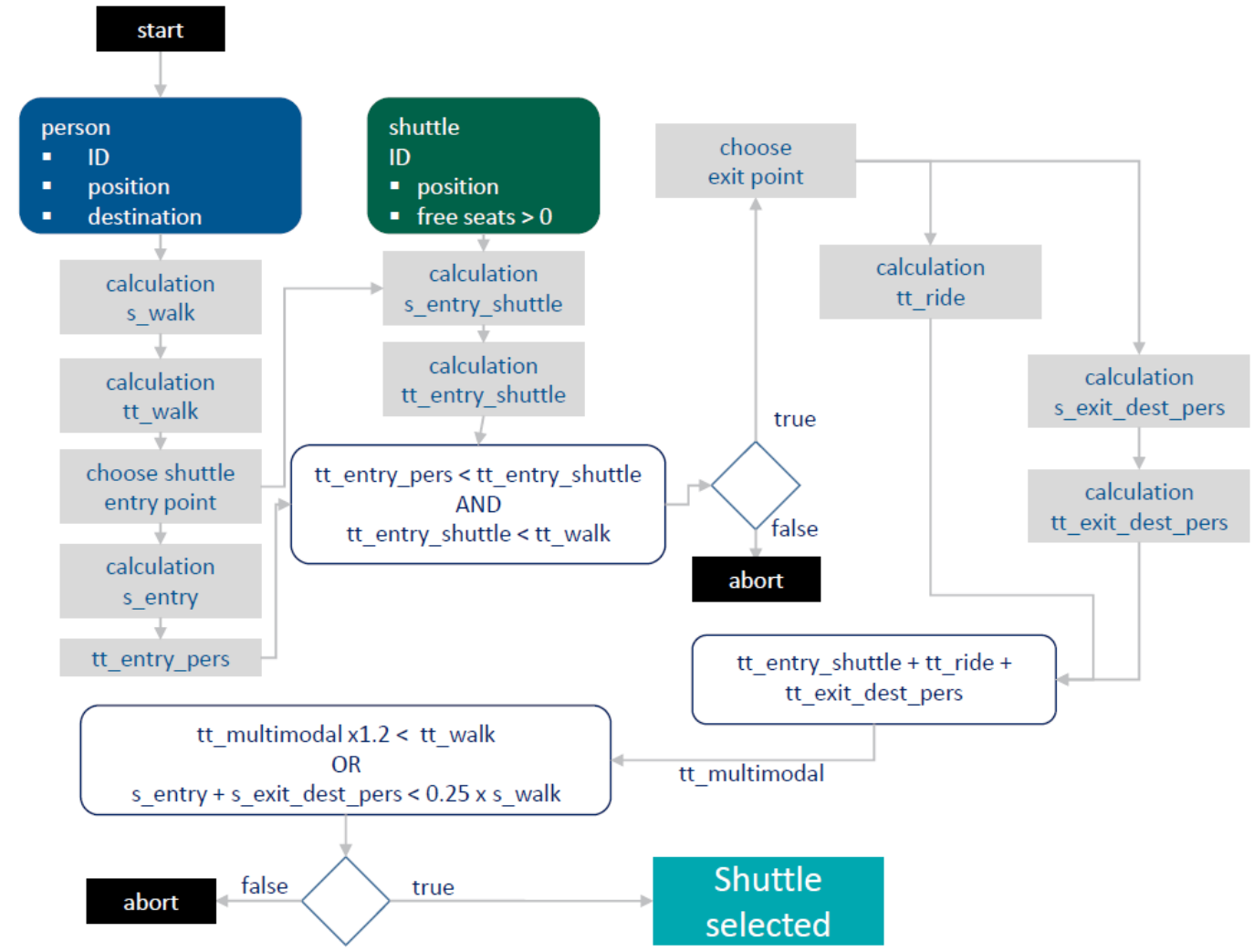

Figure 4: operation strategy for RoboShuttles in a shared space

In the last step, two constraints are checked if a shuttle is selected or not. The first constraint is that driving with the RoboShuttle saves $20 \%$ of travel time compared to the walking time to obtain a significant reduction in the travel time. The other constraint is that the walking distance in a trip with a shuttle is only $25 \%$ of the pure walking distance s_walk. If at least one of the constraints holds, a shuttle is selected. The second constraint is implemented to encounter the fact that a pedestrian will wait for a shuttle if the saving in the walking distance is high enough.

\section{Rebalancing}

After some simulation steps in the first simulations the RoboShuttles haven't been distributed equally on the route. If there have been two or more shuttles in the simulation, after some time the shuttles moved in queues. The reason is that if a shuttle stopped to pick up some passengers the following shuttle approached more and more. After some stops of the leading shuttle, the shuttles moved in a queue. If a pedestrian requests a shuttle, the first shuttle at the stop is selected. Also in case of overtaking, the queue does not resolve. The number of journeys is decreasing because the queue behaves like one shuttle. To avoid the queuing, the functionality of "rebalancing" was implemented. It is checked if two shuttles are queuing. If no passengers are on board and no trips are planned the following shuttle slows down to a velocity of $2 \frac{\mathrm{m}}{\mathrm{s}}$. With this functionality, the queuing is avoided. 


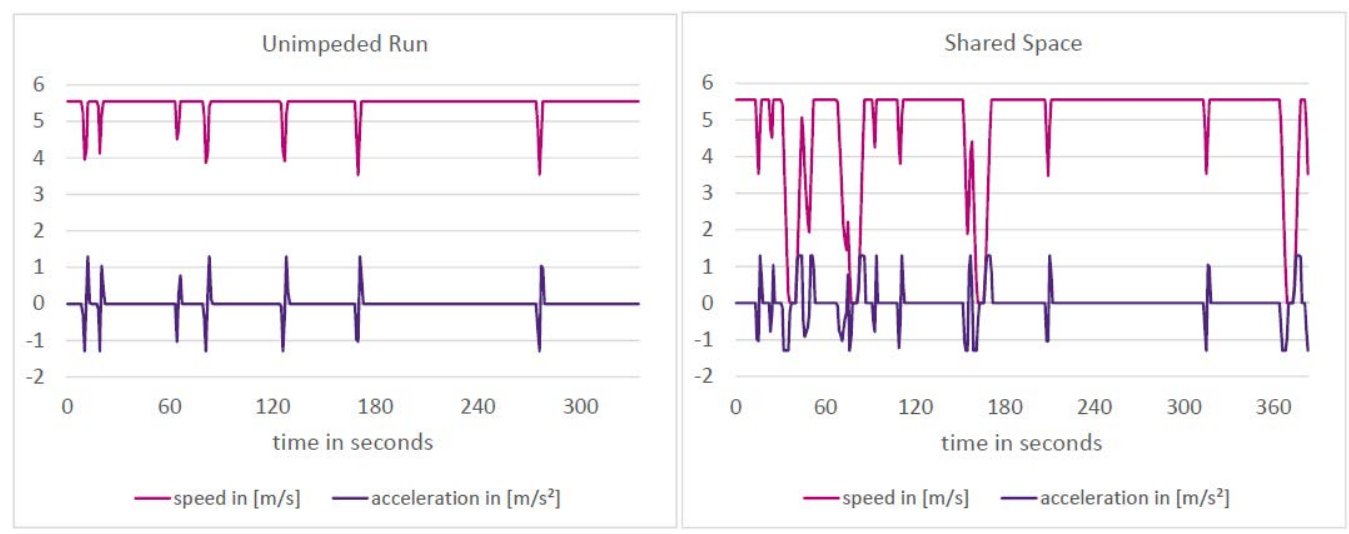

Figure 5: Velocity and acceleration over time of a RoboShuttle for the route on the research campus with and without pedestrian traffic

\section{Simulation Research Campus}

SUMO provides with TraCI a software interface to read simulation results and to control the simulation. TraCI is a TCP based client/server structure that creates a socket-server at simulation start. To improve the handling TraaS (TraCI as a Service) was developed that implements a socket-client which can be used in combination with many different clients (TraaS, 2017). In this work, TraaS was combined with Java.

Also, to improve the simulation performance the concept of subscriptions was used to retrieve the information. There are variable and context subscriptions. Variable subscriptions can be started for all parameters to observe a vehicle, e.g. velocity and position. After the start of the subscription the information of the chosen parameter is periodically send to the client. Context subscriptions are defined for the desired elements in the model. For example, a context subscription can be used to get the list of vehicles and their velocities on an edge. Because of the amount of information the use of subscriptions improved the run time of the simulation by $50 \%$.

The operation strategy was also implemented in Java. The time step size of the simulation was set to 0.1 sec. An open question was how the travel time / level of service improves with the number of RoboShuttles. Therefore, the number was varied in the steps $0,1,2,6$, and 8 vehicles, where 0 is the reference without RoboShuttles.

Also, the simulations have been done with and without rebalancing to show the improvements. The simulation was split in to the morning, when the employees travel to their working places, lunchtime, when the employees travel to the cafeteria for lunch, and the evening, where the employees leave their working places and travel to the exits of the campus. 

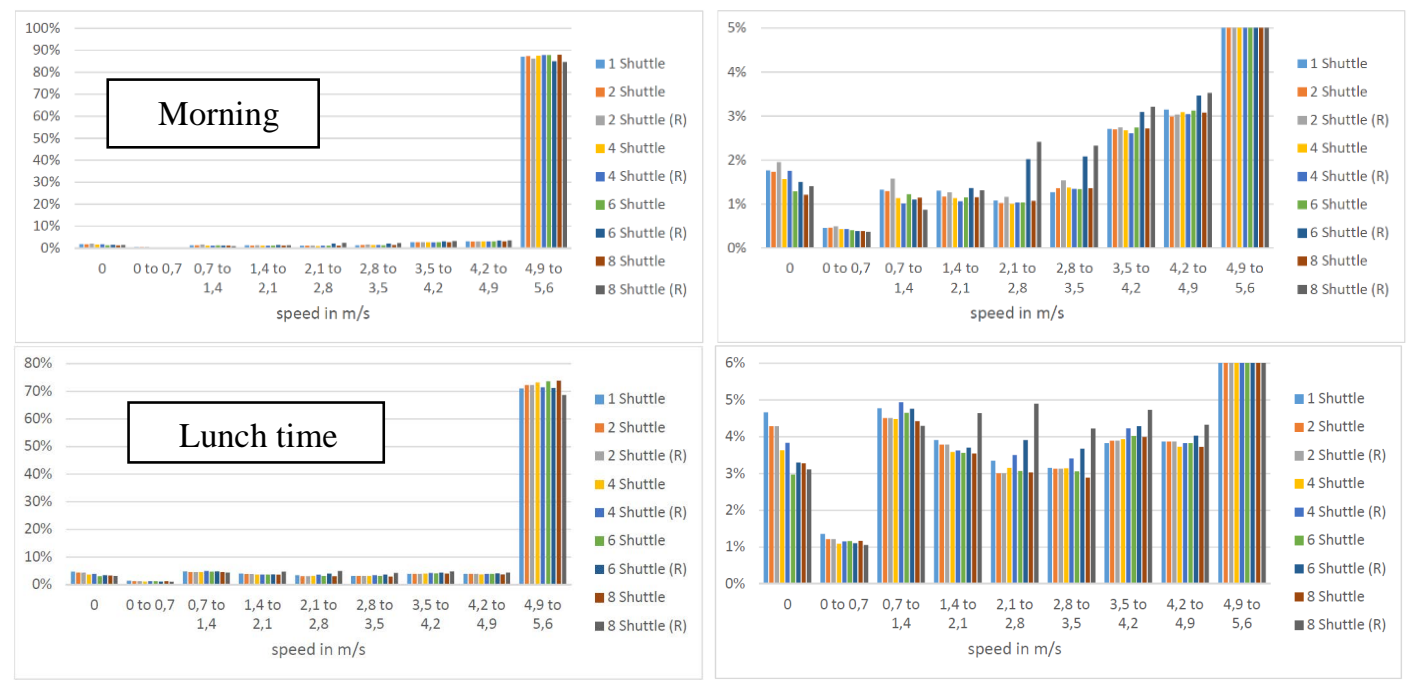

Figure 6: velocity distribution for different numbers of RoboShuttles with and without rebalancing in the morning and at lunchtime

In Figure 5, the velocity and acceleration of two different RoboShuttle trips are shown. In case of the free route the velocity is never less than $3.5 \frac{\mathrm{m}}{\mathrm{s}}$. The mean value of the velocity is higher than in the case of the shared space simulation. In both cases the acceleration stays in the boundaries of $\pm 1.3 \frac{\mathrm{m}}{\mathrm{s}^{2}}$.

In case of the shared spaced, the RoboShuttle brakes to still stand four times because of stops so that pedestrians can board or exit the RoboShuttle.

Also, the greater variation in the speed profile is caused by the interaction with pedestrians. The RoboShuttle has to overtake pedestrians or in case where not enough space is available, the RoboShuttle has to decelerate.

In Figure 6, the velocity histogram is shown in the morning for different numbers of RoboShuttles on the campus. The " $\mathrm{R}$ " stands for rebalancing. It can be seen that the RoboShuttles drive most of the time with the maximum velocity because in the morning only a few pedestrian are walking on the campus. To get a closer look at the velocity distribution at lower values the $\mathrm{y}-$ axis is bounded to $5 \%$. The distribution of the velocity has a peak at $0 \frac{\mathrm{m}}{\mathrm{s}}$ because of the stops for the boarding and exit of the passenger. For the results in case of the rebalancing with 6 and 8 shuttles the distribution between 2.1 and $4.9 \frac{\mathrm{m}}{\mathrm{s}}$ is greater than in the other cases. The shuttles have to brake and accelerate more often for a homogeneous distribution of the shuttles on the research campus. But the rebalancing leads to a higher level of service as can be seen in the travel time of the pedestrians in Figure 7. 


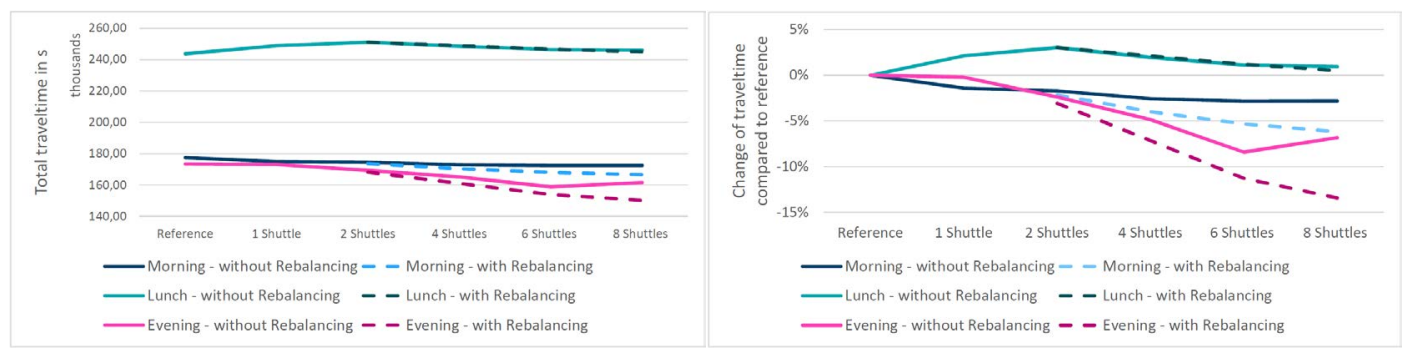

Figure 7: Travel time of the pedestrian on the research campus

The velocity distribution at lunchtime is different from the distributions in the morning and the evening because of the high pedestrian traffic flow to the cafeteria, which causes a high interference of the RoboShuttles. Compare with Figure 6.

As mentioned above, the distribution of the overall travel time improves with the number of RoboShuttles, compare with Figure 7. The rebalancing yields further improvements. But for individual people, the travel time increases because a planned shuttle ride cannot be reached and therefore the waiting time increases. The increase of the travel time at lunchtime with the number of RoboShuttles can be explained by the increased pedestrian traffic flow on the campus and a higher interference of the RoboShuttles, and therefore the travel time estimated in the operation strategy is not correct, which causes longer waiting times. A better estimation of the travel time of the shuttles would lead to an improvement.

For the requirements of a powertrain, the number of start and stops is an interesting measure. Figure 8 shows the number of braking events depending on the number of shuttles and rebalancing. Also the share of the stop events is shown. The number of RoboShuttles has only a small influence on the number of brake events. The number of brake events at lunchtime is the highest because of the pedestrian traffic flow to the cafeteria. Rebalancing yields a small decrease in the number of brake events. But the share of the stop events decreases with increasing number in RoboShuttles, and rebalancing also. The scenario in the evening has the highest decrease in the number of stop events. The number drops from $22 \%$ to $12 \%$.

\section{Conclusion}

A simulation scenario for a shared space with pedestrians and RoboShuttles was set up in SUMO. The scenario consists of pedestrian and vehicle traffic, where the pedestrian traffic is modelled with poison distributions. The vehicle traffic is defined by the RoboShuttles on a fixed route. Furthermore, a multi modal travel time minimization strategy was implemented to plan the trips of the pedestrians with the RoboShuttles. Because of the buildup of queues, rebalancing was invented so that a better distribution of the RoboShuttles on the campus is obtained. The simulation results show an improving of the travel time for the pedestrians in the morning and evening. No improvements in travel time is obtained at lunchtime because of the great pedestrian traffic.

Altogether, it could be shown that the simulation of multi modal trips of pedestrians in SUMO is possible, and the traffic system "shared space” could be studied. 


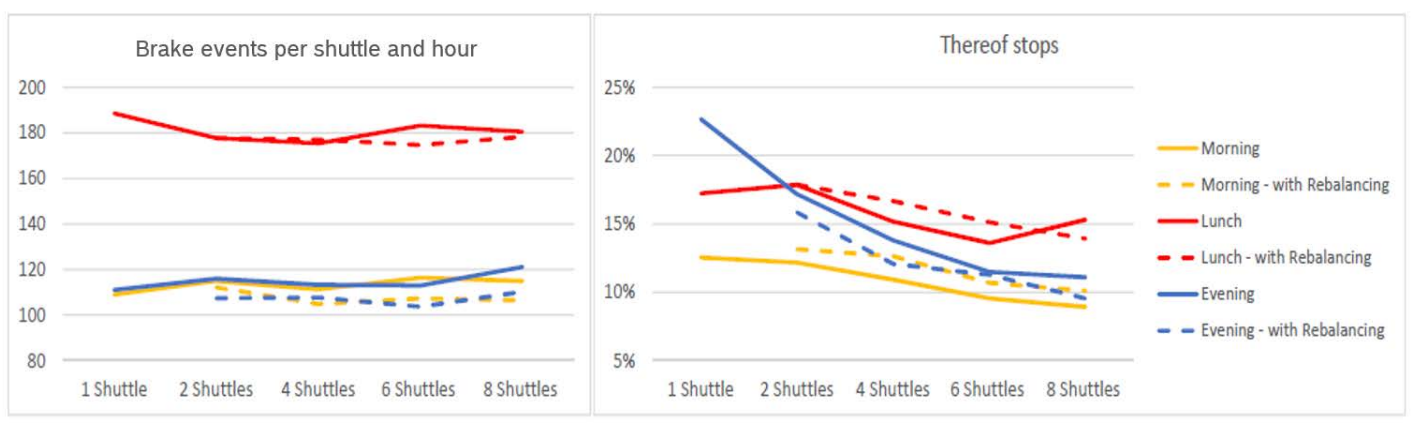

Figure 8: Number of brake and stop events depending on the number of shuttles and rebalancing

\section{References}

A. Neumann, S. K. (2014). Boarding and Alighting Time of Passengers of the Berlin Public Transport System. http://www.vsp.tu-berlin.de/publications.

AURO. (2017). Re-Inventing Campus Mobility. http://auro.ai/.

CITYMOBIL2. (2016). CityMobil2 - Experience and recommendations. http://www.citymobil2.eu/en/upload/Deliverables/PU/CityMobil2\%20booklet\%20web\%20fi nal_17\%2011\%202016.pdf.

Daniel Krajzewicz, J. E. (2012). Recent Development and Applications of SUMO - Simulation of Urban MObility. International Journal On Advances in Systems and Measurements, 128-138.

Deutsche Bahn AG. (2017). Deutsche Bahn setzt mit ioki erstmals autonom fahrenden Bus im öffentlichen Linienverkehr ein. https://www.dbregio.de/db_regio/view/zukunft/autonomesfahren.shtml.

EASYMILE. (2017). Shared driverless transportation for the last mile. http://easymile.com/.

IVI, F. (2017). Synchronized Mobility. . https://www.synchronemobilitaet.de/en.html.

Marshall, A. (2017). Don't Look Now, But Even Busses Are Going Autonomous. https://www.wired.com/2017/05/reno-nevada-autonomous-bus/.

NASA. (kein Datum). SRTM Mission. https:/www2.jpl.nasa.gov/srtm/.

OPENSTREETMAP. (2017). OPENSTREETMAP. http://www.openstreetmap.org/about.

OSMFILTER. (2017). OSMFILTER. http://wiki.openstreetmap.org/wiki/DE:Osmfilter.

Tor, J. (2017). Ambitious, University-led effort explores mobility technologies. https://www.unr.edu/nevada-today/news/2017/intelligent-mobilitylaunches.

TraaS. (2017). TraCI as a Service. http://traas.sourceforge.net/cms/.

WAYMO. (2017). https://waymo.com. 